\title{
MAP - A Mobile Agile Printer Robot for on-site Construction
}

\author{
Julius Sustarevas ${ }^{1}$, Daniel Butters ${ }^{1}$, Mohammad Hammid ${ }^{1}$, George Dwyer ${ }^{1}$, Robert Stuart-Smith ${ }^{1} 2$ \\ and Vijay M. Pawar ${ }^{1} 2$
}

\begin{abstract}
In this paper, we present a Mobile Agile Printer (MAP) construction robot; a highly agile, 4-legged, omnidirectional robot capable of 3D printing large structures. To overcome dynamic challenges when operating within an outdoors construction site, MAP incorporates a high-DoF 3D printing system connected to a mobile platform with novel features designed to enable disturbance rejection and live adaption to the robot's pose. In doing so, we demonstrate the benefits of designing construction robots with a focus on agility, a compact working volume and ability to operate within a potentially unlimited workspace. Performance tests were conducted showing smooth omni-directional motion as a key requirement for maintaining low 3D printing trajectory deviations over a large volume. In doing so, we show that MAP has the ability to construct in new ways more sensitive to its environment, context and concurrent on-site operations.
\end{abstract}

\section{INTRODUCTION}

Compact and agile fabrication robots have the potential to create a paradigm shift in the construction industry. By combining technological progress in both mobile robotics and additive manufacturing, we can begin to explore new capabilities for on-site 3D printing of buildings; achieving a future whereby the next generation of construction sites are fully autonomous. To deliver a step change in productivity, cost reduction, sustainability and managing hazards involved in construction [1], we aim to investigate new robot designs capable of adapting to dynamic building site environments. Currently, few studies exist that use robots with small form factors and limited spatial impact that are agile enough for large-scale outdoor 3D printing. To demonstrate the utility of this approach, in this paper we present MAP- a novel omni-directional mobile platform with a high Degree of Freedom(DoF) robot arm capable of large-scale on-site construction. We illustrate the functionality of the robot by evaluating its design for greater mobility and supporting enhanced construction capabilities afforded by $3 \mathrm{D}$ printing technologies. Through this study, we aim to demonstrate the benefits of designing construction robots with a focus on agility and mobility to build in new ways that are more sensitive to their environmental context.

The construction industry relies on manufacturing methods that combine off-site and on-site fabrication. With more than 3100 units estimated to be sold between 2018-2020,

\footnotetext{
${ }^{1}$ Authors are members of the Autonomous Manufacturing Laboratory within the Department of Computer Science, University College London, Gower Street, WC1E 6BT, UK. julius.sustarevas.16@ucl.ac.uk daniel.butters.160ucl.ac.uk

2 Corresponding authors: r.stuart-smitheucl.ac.uk and v.pawar@ucl.ac.uk
}

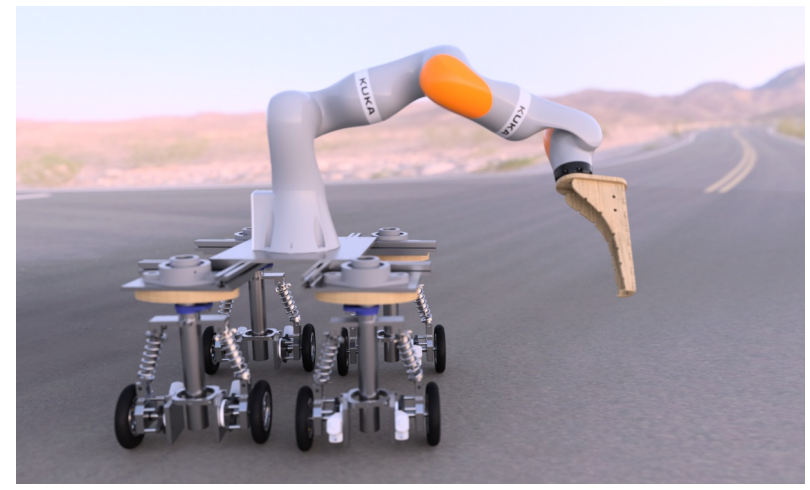

Fig. 1. MAP construction robot - Four-Legged, Mobile Agile Printer Robot for on-site Construction

robots have been involved in off-site fabrication for some time[2]. Nonetheless, the completion of every building also involves partial or full on-site construction which remains to be heavily reliant on manual labour and large-scale construction machinery. Within this context current methods are inflexible, unproductive and statistically one of the most dangerous manufacturing environments, with three times the average rate of fatalities compared to other industries in the UK [3]. Unlike factory environments where work zones demarcate workers away from dangerous machinery, on-site construction methods operate at scales that cover the entire work space thus making multi-tasking extremely hazardous and difficult to implement safely.

To integrate autonomous construction machinery within the fast-paced, congested and hazardous environment of construction sites it is necessary to develop agile mobile robots capable of undertaking manufacturing tasks with greater DoF and mobility, whilst also reducing the space these robots occupy. Mobile robots have been proposed for discrete activities such as brick layering [4] [5] however these platforms have limited DoF and agility that constrain their ability to dynamically engage with other robots, people or construction machinery. Expanding this further, greater mobility through distributed robotics can also provide greater scope for creativity. Resembling the process termites used to construct their mounds, a decentralized network of robots operating as a multi-agent system is capable of collectively building structures, designing through individual actions that are locally reactive to the environment. In this capacity, design may be an emergent property of autonomous robotic construction. Harvards TERMES project demonstrated such capabilities utilizing bespoke robots and building blocks [6], 
displaying a capacity to assemble structures using a collective of simple independent autonomous robots. Key features include the wheel design for climbing and motion using differential drive. Another example includes V. Kumars group showing a team of unmanned aerial vehicles constructing a pre-designed space frame [7]. A novel multi-robot solution for concrete $3 \mathrm{~d}$ printing is provided in IAAC's MiniBuilders project[8]. IAAC's Single-Task based approach consists of a heterogeneous group of robots that are each specifically designed for a single construction task. Current tested robots include: a foundation laying robot that prints the initial layer of concrete; a wall printing robot that attaches itself to top edge to deposits a concrete roof; and a surface printing robot that can be attached via suction. Other small-scale systems include Droplets [9], that also implement omni-directional motion using vibration motors and thus benefit from vector based motion planning. Altogether, and common with most swarm and multi-agent systems, omni-directionality is an essential feature supporting smooth continuous motion to aid robot interaction and motion planning.

In this paper, we further construction scale $3 \mathrm{D}$ printing by addressing the challenges of building site terrain, large working volume and robust deposition of material. We propose a mobile manipulator printing approach that includes a compact mobile omni-directional robot with a high DoF 3D printing system that can adapt to its terrain and extrude building materials along trajectories far greater in length than the confines of the robots own work envelope. The proposed system addresses the scale, print volume and robustness requirement by being designed to be a unit of a multi-robot system. To achieve this, it is necessary to have sufficient DoF to compensate for variations in terrain to achieve pose accuracy relative to state of the art localisation methods, and to integrate vehicle and printing motions within a common control system. We illustrate the functionalities of the robot design and evaluate its utility in supporting construction via 3D printing building technologies. In doing so, we aim to demonstrate the benefits of construction robots with a focus on agility and mobility to build in new ways that are more sensitive to their environment and concurrent site operations.

\section{BACKGROUND AND RELATED WORK}

Whilst progress has been made in autonomous construction systems in controlled laboratory settings, relatively little work exists using these methods in outdoor environments. Within this context, we explored additional requirements that include: 1) greater mobility to operate within a potentially unlimited workspace and 2) building mechanisms that can adapt to the dynamics and variability to unknown environmental conditions.

\section{A. Large-scale Omni-directional Drive Mechanisms}

To achieve greater mobility and utilise distributed robotic principles, a desirable requirement is to support omnidirectionality. From mechanum to omni-wheels, a number of studies have investigated different designs capable of achieving smooth motion, high torques and payloads [10]
[11]. Examples of state of the art autonomous construction systems can be found in a variety of commercial such as those developed by Kuka. However, as discussed by Aoki et al, a key characteristic of an omni-wheel is that the axis of rotation needs to be parallel to the contacting floor in addition to the attached rollers for smooth motions [12]. This is a significant drawback when considering its use in multiterrain environments where the contact between the wheels and the surface is unknown or cannot be guaranteed.

Another approach is synchronous drive. This involves three or more wheels being independently driven and their orientation with respect to the robot actively controlled. This approach often uses a chain and gear mechanism accompanied by a single motor to control the orientation of all wheels at once [13]. This way it is assured that they all point in the same direction. In some systems, the wheels are also all controlled by a single motor, which allows for omni-directional motion on a plane using only two actuators. However, systems like this are not truly holonomic as in any point in time system constraints prevent instantaneous motion along direction perpendicular to wheel direction. Other types of wheels, enabling the omni-directional traveling have been developed that demonstrate conditions similar to using rubber tires and have a greater tolerance for rough surface [14].

One theme that often emerges is the need for robot reconfigurability where it can actively change the geometry of how it is interacting with the ground and achieving traction or contact. For outdoor environments an alternative to wheels is to use tracks. Whilst tracks allow for great traction on a variety of surfaces, they do not lend themselves well to omni-directionality [15]. At present, there are limited implementations of omni-directional systems that use tracks for rough terrain. Except for screw-drive design[16], all reviewed solutions used synchronous drive. A successful commercial shepherding robot, designed to roam far and be tolerant of grass and wet terrain is SwagBot [17]. This robot is capable of high speed and high pulling force. It uses synchronous drive to achieve omni-directionality via its single wheeled legs and dedicated motors for rotating the wheels. Further studies show that the compliance and flexibility of the robot, as well as use of compliant rotating legs are important factors. This ensures the robot can distribute its weight on uneven ground, perturbed at the scale of the robot. With respect to the transportation of heavy materials, having leg compliance will ensure robustness of the system.

The closest example that match our requirements is the active split offset caster (ASOC) design put forward by MIT researchers [18]. This system uses offset dual wheels to rotate the leg, which is on a passive joint, and achieve synchronous drive. The wheel pairs are on a passive joint with suspension perpendicular to the leg providing increased traction and contact. An evaluation of this design has shown that it prefers all directions of motion equally, suitable for supporting omni-directional movement. The design includes power supplies located in the legs, hence making the legs autonomous modules, containing their own computational power for motor control and power supply. The centre of 
the robot contains the computational unit for motion planning and higher-level control, radio frequency communication is used to communicate with the ASOC leg modules. The designs described in this subsection are summarised below:

\begin{tabular}{|c|c|c|}
\hline Drive & Strengths & Weaknesses \\
\hline Omni-wheels & Holonomic & Rough terrain \\
\hline Synchronous & Rough terrain, speed & Not holonomic \\
\hline Tracks & Rough terrain & Not omni-directional \\
\hline ASOC & Rough terrain, speed, holonomic & Complexity \\
\hline
\end{tabular}

Fig. 2. Summary of overviewed drive methodologies.

\section{B. High-DoF Additive Manufacturing}

Already, within the field of additive manufacturing, researchers and industry are demonstrating a significant reduction in material use, cost and waste involved in building. At present, the construction industry is starting to explore additive manufacturing techniques with gantry 3D printers [19] [20]. Due to their scale, these systems are mostly utilised off-site and hard to reconfigure to the geometries typically found in outdoor construction sites. Further, as their large work envelope propagates across multiple work zones, this prevents parallel operations from taking place as per established industry methods that requirement a diverse number of dynamic activities and space-sharing relations. Alternatively, researchers are also investigating flexible printing platforms with higher DoFs such as a robot arm. Zhang et al. documented the utility of robotic arms in several direct metal systems [21]. Multiple studies have shown high DoF systems also improve material usage in Directed Energy Deposition Additive Manufacturing processes (a full review found in [22]). Singh and Dutta have also shown that by changing the build direction throughout the part can improve finishing quality [23]. Further, Keating and Oxman have demonstrated a material extrusion platform using a $6 \mathrm{DoF}$ arm that allowed printing of overhangs without the need for support by changing the orientation of the part relative to the deposition nozzle [24]. These studies show that the use of high DoF systems provides the potential for better control of the building process and adaptation to the environment.

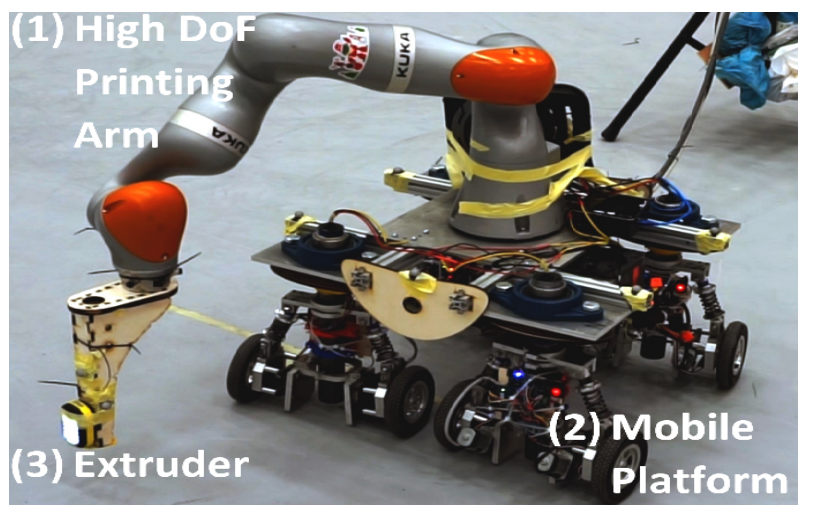

Fig. 3. Mobile printer - consists of three parts: 1) a 4-legged mobile platform 2) high DoF robot arm suitable for adaptive 3D printing motions and 3) an end-effector material deposition mechanism

\section{MOBILE AGILE PRINTER ROBOT}

To embody the proposed design, an omni-directional platform carrying a high-DoF robot arm was constructed as seen in Figure 1. Before these two most significant components are discussed in detail, system-wide overview is provided.

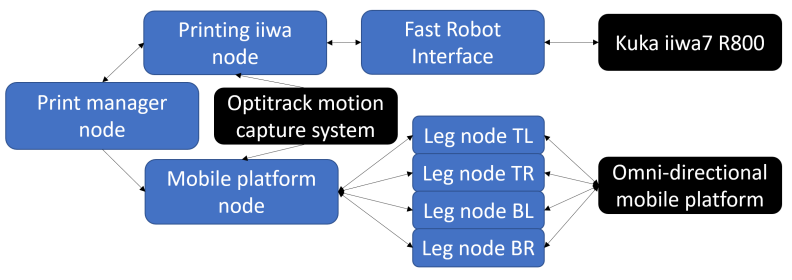

Fig. 4. Mobile printer abstract diagram showing ROS nodes in blue, major hardware components in black

The entire system uses Robot Operating System (ROS) middleware. The node that coordinates the high level functioning of the mobile platform and the printing high-DoF robot arm (Kuka LBR iiwa7 R800) is the Print Manager node. The basic function of this node is to implement a queue data structure and manage print layers, that are generated before printing, and consist of target poses for the extruder and the base. New poses from the print layer are added to the print queue as old points are printed. For every target extruder pose, the mobile platform has a corresponding target pose to reach. The manager node maintains a print queue of length 5 for the printing robot arm and commands the mobile platform to first corresponding platform pose.

A print target pose is considered to be reached when the distance from the end effector to the point is less than 2.5 $\mathrm{mm}$. When the printing iiwa node reports that it has reached the first pose in the print queue, that pose is removed and a new pose is added to the print queue from the layer. The corresponding base target pose is updated, and the printer continues through the layer. This control node allows the mobile printer to act in a similar way to a conventional 3D printer, as a trajectory is set to play out on the system, and can be paused and resumed at the same point in the trajectory. Only a small portion of the trajectory is sent to the printing robot arm at any time, and this provides potential for new functionality to be added in future iterations.

To isolate the evaluation of control methods and effects of proposed design on print quality and function, a few placeholder systems were used. A 24 camera Optitrack PRIM17W [25] motion capture was used for robot positioning. This system offers sub-millimeter accuracy motion tracking over a 10 meter diameter workspace. Also a tether was used to deliver power and robot arm control from external sources. Furthermore, whilst the arm end effector and extruder are used as interchangeable terms, implementation of an extruding system is left for future research.

\section{A. 4-Legged Omni-directional Mobile platform}

To implement the desired characteristic of omnidirectionality, the mobile platform was built as a four-legged platform. Each leg is on a passive revolute joint which is 
actuated via a two-wheel differential drive at the bottom of the leg. This design is inspired by the ASOC module [18]. However, offset caster wheels give rise to asymmetry that can cause a reduction in stability of the base, in addition to affecting the printing arm workspace. Therefore, wheel and leg rotation axes were designed to intersect.

To demonstrate that omni-directionality was implemented successfully, firstly the mathematical model of the mobile platform was derived, the system implementation presented and omni-directionality of platform assessed.

1) Mathematical model: The state of the mobile platform is considered to be in its two dimensional position $(x, y)$, its orientation $\psi$ and orientations of its four legs $\theta_{l}$ where $l \in$ legs $=\{T L, T R, B L, B R\}$. Hence $\overrightarrow{\mathbf{x}}=$ $\left(x, y, \psi, \theta_{T L}, \theta_{T R}, \theta_{B L}, \theta_{B R}\right)^{\top}$.

Considering the four legs as point velocity sources $\vec{v}_{l \in l e g s}$. This immediately provides an expression for the derivatives of first three elements of the state:

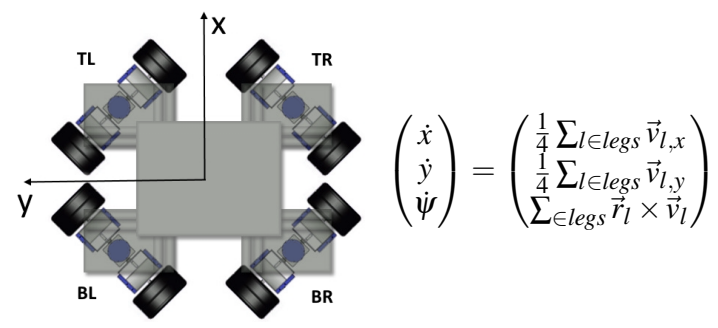

Fig. 5. Left: Mobile platform diagram showing its default $X$ configuration, its frame of reference and identifying Top Left, Top Right, Bottom Left and Bottom Right legs.Right: Mobile platforms lateral and orientation derivatives

Here the $\vec{r}_{l}$ denotes the vector from the center of the base to the leg 1. Finally, using formulas of differential drive, full state derivative can be expressed in terms of the input of the system, which in this case is wheel angular velocity.

$$
\dot{\overrightarrow{\mathbf{x}}}=\left(\begin{array}{c}
\dot{x} \\
\dot{y} \\
\dot{\psi} \\
\dot{\theta_{T L}} \\
\dot{\theta}_{T R} \\
\dot{\theta}_{B L} \\
\dot{\theta}_{B R}
\end{array}\right)=\left(\begin{array}{c}
\sum_{l \in l \text { egs }} \frac{R}{2}\left(\omega_{l, R}+\omega_{l, L}\right) \cos \left(\theta_{l}\right) \\
\sum_{l \in l \text { legs }} \frac{R}{2}\left(\omega_{l, R}+\omega_{l, L}\right) \sin \left(\theta_{l}\right) \\
\frac{R}{2} \sum_{l} \vec{r}_{l} \times\left(\omega_{l, R}+\omega_{l, L}\right) \hat{v}_{l} \\
\frac{R}{d}\left(\omega_{T L, R}-\omega_{T L, L}\right) \\
\frac{R}{d}\left(\omega_{T R, R}-\omega_{T R, L}\right) \\
\frac{R}{d}\left(\omega_{B L, R}-\omega_{B L, L}\right) \\
\frac{R}{d}\left(\omega_{B R, R}-\omega_{B R, L}\right)
\end{array}\right)
$$

Here $\hat{v}_{l}=\left(\cos \left(\theta_{l}\right), \sin \left(\theta_{l}\right), 0\right)$, the unit vector direction of leg orientation. $R$ is the radius of the wheels and $d$ is separation. $\omega_{l, L}, \omega_{l, R}$ are left and right wheel angular velocities.

2) Implementation: On top of omni-directionality, a number of other desired characteristics of the mobile platform were implemented. The mobile platform was build with laser cut aluminium parts and eight $9 \mathrm{Nm}$ torque motors, which led to the robot having a carrying capacity of over $70 \mathrm{Kg}$. Capacity of this order of magnitude, or higher, is seen as desirable as it enables carrying material deposition equipment, like clay extruders, and enough material for trajectories greater than manipulators immediate workspace. The platform employs five independent computational units running ROS that communicate via a wireless network: Intel $\mathrm{Nuc} 7$ mounted on the central platform and Raspberry $\mathrm{Pi}$ zero $W$ on each of the legs. This makes each of the legs computationally independent as only DC power is passed through the revolute joint connecting the leg.

As a result of having multiple networked computing units and the symmetry in the motion model, the control strategy adopted is hierarchical, namely three cascaded PID controller as shown in Figure 6 below.

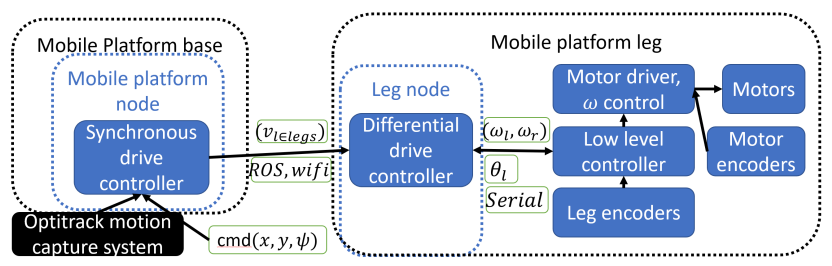

Fig. 6. Mobile platform control hierarchy. Major control components shown in blue, ROS nodes in dotted blue, base-leg hardware separation in black dotted, control commands and medium in green. The mobile platform leg component is repeated for each leg.

The motor driver, Parallax DHB10, implements PID control by receiving feedback from motor encoders and controlling for motor angular velocity. The leg node implements a differential drive controller. This uses a PI controller to follow a desired leg orientation target using feedback from leg orientation encoders. Note that the serial communication between a low-level controller (Arduino Uno) is bi-directional, as the low-level controller registers encoder readings and sends them to the leg node. Finally, the mobile platform node implements a synchronous drive controller that commands desired leg velocity vectors according to the received desired position and orientation of the whole platform. Position and orientation feedback from the Optitrack system is used by this controller. Note that this synchronous drive controller is not holonomic and uses a state machine to switch between position and orientation control, therefore having same drawbacks as conventional synchronous drive.

3) Evaluating omni-directionality: The ASOC module was designed to have perfect kinematic isotropy of 1. As laid out by authors of ASOC this is a condition in which a robot possesses a constant input velocity/output velocity ratio for all possible output velocity directions [18]. This measure can be derived as the ratio of the smallest to largest eigenvalues of the jacobian matrix [26]. For the mobile platform, the default leg configuration is when all legs face away from the center of the base at a $\frac{p i}{4} \mathrm{rad}$ angle. From the equation 1 we see that linearizing the system at default $\theta_{l}, l \in$ legs would mean the first two rows become identical hence leading to kinematic isotropy of 1 . This is because such leg configuration restricts the movement of the base and all directions of motion are equally not preferred. The default leg configuration offers advantages in stability as it optimally spreads out all 8 points of contact of mobile base with the ground. Therefore, this configuration is chosen to be adopted whenever the base is stationary. Furthermore, this configuration provides least rotation time for wheels to align for motion in any direction. 
To empirically evaluate the omni-directionality of the mobile platform two experiments were conducted. Firstly, as synchronous drive relies on the leg orientation control to be reliable, the step input response to leg orientation control is presented below in figure 7 .

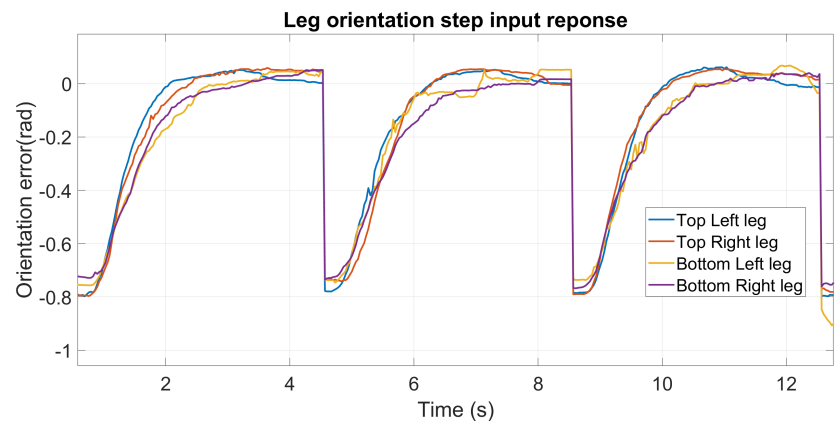

Fig. 7. Leg orientation control response to $\frac{p i}{4}$ step inputs for each leg.

Data was gathered for 10 trials of responses to $\frac{p i}{4}$ and $\frac{p i}{2}$ step inputs. The data was cropped to illustrate the response of each leg. For both series of trials a response time to settling to $0.1(\mathrm{rad})$ was computed together with error when settled:

\begin{tabular}{|c|c|c|}
\hline & Error when settled & Response time \\
\hline 45 degree steps & $0.0329 \mathrm{rad}, \mathrm{std}=0.0273$ & $1.4297 \mathrm{~s}, \mathrm{std}=0.2475$ \\
\hline 90 degree steps & $0.0124 \mathrm{rad}, \mathrm{std}=0.0367$ & $1.5397 \mathrm{~s}, \mathrm{std}=0.482$ \\
\hline
\end{tabular}

Whilst data shows the response time of leg orientation to be only about $1.5(s)$, the legs remain synchronized hence allowing synchronous drive to move the platform in any direction. To verify that the direction of motion response is consistent throughout all directions, an experiment was set up that tested the target velocity vector direction against the achieved one. During this experiment the platform was

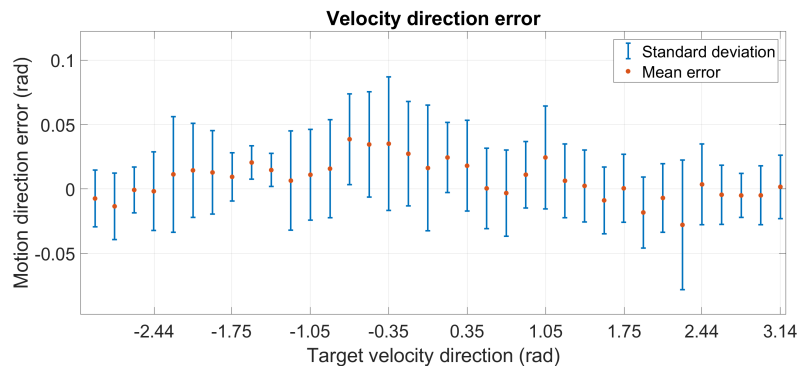

Fig. 8. Error in direction of motion over a 3 second sustained motion in every direction.

commanded to move in every direction in intervals of $10^{\circ}$. The direction of motion was maintained for 3 seconds. Figure 8 illustrates the error between direction of motion over this interval and the target direction of motion.

It can be seen that the discrepancies in error between different target directions are insignificant therefore empirically showing that the platform does not have a preferred direction of motion and is omni-directional.

\section{B. High-DoF Printing Robot Arm}

The robot mounted to the mobile platform is the 7 degree of freedom KUKA LBR iiwa 7 R800. The arm carries a pay- load of $7 \mathrm{~kg}$, has a maximum reach of $800 \mathrm{~mm}$ and weighs $23.9 \mathrm{~kg}$. The arm is capable of multiple configurations for most end effector poses due to its 7 degrees of freedom, and has a repeatability of $\pm 0.1 \mathrm{~mm}$. This arm is designed to be safe for use near humans, with compliant control and torque measurement systems that shut down the robot when an external torque is detected by the arm.

The printing robot arm is controlled with the Fast Robot Interface (FRI). This interface is part of KUKA Sunrise OS, and allows real time access to the robot joint position controller from an external computer [27]. The FRI can command the joint positions of the robot at a rate of $500 \mathrm{~Hz}$. The interface is sent joint positions by the position controller from the ROS Control packages [28].

Motion planning is carried out using the Moveit Motion Planning Framework [29]. This is an open source framework built into ROS that allows a high degree of customization, as developers can write their own plugins to enhance the capabilities of the software. The printing arm node uses RRTConnect [30] to sample kinematic solutions to the goal trajectory, then checks for any collisions within the planning environment. If a valid plan is found, the joint trajectory is sent through the FRI to the ROS position controller, which sends the trajectory to the KUKA.

A plugin for the Moveit Interface was developed to smoothly overwrite the trajectory that the printing robot arm is currently executing, so that the system can replan while it is executing a previous plan. This capability means that the printing robot arm can change course at a rate of up to 50 $\mathrm{Hz}$ to account for outside disturbances, such as the mobile platform changing orientation. This control loop implements a queue data structure for the next 5 points in the printing trajectory. The system aims to prevent steady state error by only adding a new point to the print queue when the extruder has reached the first point in the queue.

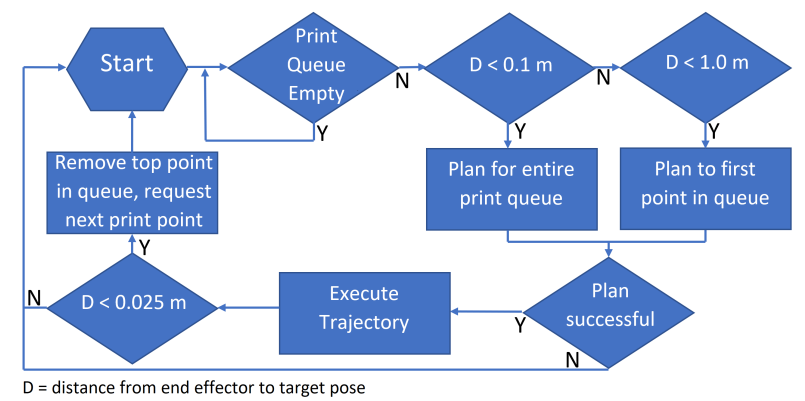

Fig. 9. High level flow diagram of the control loop for the printing robot arm

Figure 9 shows the high level control loop for the printing robot arm. This consists of waiting until the printing robot arm can safely reach the first point in the printing queue, to then moving to that point. When the end effector is close enough to start printing, the printing arm plans a motion through the whole print queue, and extrusion is activated. The motion plan is continually updated with the latest position of the mobile platform so that the arm can account for any 
disturbances. When the first point in the queue is reached, this is removed from the queue and the next one is added until the entire layer has been printed. This continues as new layers are pushed to the printing iiwa node.

It is important for the extruder to follow the print trajectory accurately. In gantry based extrusion systems, this is often achieved with open loop control as the print trajectory is restricted to the workspace of the gantry. However, in this system the workspace of the entire system is larger than the limits of the maximum reach of the printing robot arm. Therefore, a positioning system is required to determine when the print trajectory comes within reach of the extruder. The Optitrack motion capture equipment was used to track the pose of the mobile platform at $120 \mathrm{~Hz}$. This is used for manipulator planning in the Moveit environment. The base follows its own independent trajectory, and the arm will plan movements to counteract that motion hence following printing trajectory. This is a method for motion compensation, and the performance is evaluated in the following paragraphs.

The experiment was set up so that the printing robot arm would re-plan to a set point in the center of the workspace at a rate of $50 \mathrm{~Hz}$. The mobile platform would then introduce a disturbance to the system by moving along one axis in a sinusoidal motion. The disturbance introduced was constant in amplitude but varying in period of oscillation. The period of oscillation varied from 6 seconds to 42 seconds, and the amplitude was $0.3 \mathrm{~m}$ in $\mathrm{X}$ axis (sideways with respect to the robot orientation) and $0.1 \mathrm{~m}$ in $\mathrm{Y}$ axis (forward with respect to the robot orientation). The position and orientation of the mobile base and extruder were recorded at $120 \mathrm{~Hz}$ using the Optitrack motion capture software for analysis.
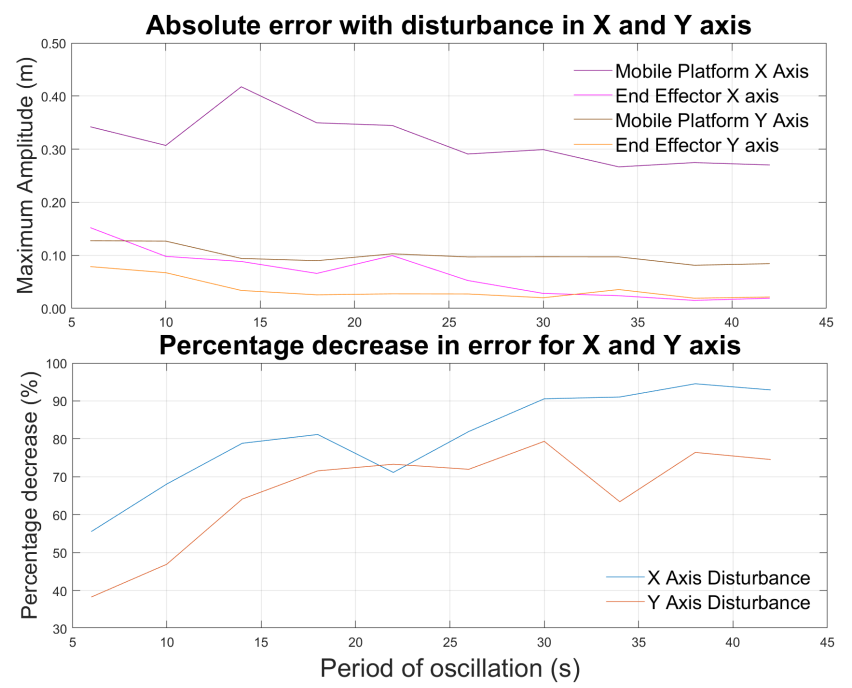

Fig. 10. Amplitudes of oscillation of the mobile platform and end effector and percentage decrease in error for end effector

Figure 10 shows the maximum distance from the set point that the extruder reached when being disturbed by the base motion. The amplitude of the disturbance is constant throughout the experiment, so as the period decreases, the velocity of the base will increase. For all periods of oscillation, the extruder has a significantly lower maximum amplitude than the mobile base. This suggests that the printing arm node is planning motions that compensate for the movements of the mobile base. The percentage decrease in error for $\mathrm{X}$ axis disturbances ranges from $55.54 \%$ to $92.91 \%$, and for $\mathrm{Y}$ axis oscillations, the percentage decrease in error ranges from $38.28 \%$ to $74.55 \%$ with a period of 6 seconds and 42 seconds respectively. This suggests that the motion compensation performs better with oscillations at a lower frequency. This is expected, because at lower frequencies the base moves at a lower velocity. This result also suggests that a lower printing velocity will produce higher accuracy prints.

\section{PRinting EVAluation}

To evaluate how well the proposed design lends itself to 3D printing for construction, the printing trajectory error as well as feasible printing strategies were investigated. The print trajectory error was computed by tracking the extruder using the Optitrack system. Firstly the printing iiwa node was assessed when the mobile platform was stationary - allowing for comparison against simultaneous motion printing. Then a multi-phase experiment was set up to illustrate a subset of possible trajectories that when the compact arm workspace is moved by as mobile platform during printing.

\section{A. Stationary printing - Spiral}

To evaluate the printing accuracy with a stationary base, a spiral geometry was used with no platform motion. This trajectory is part of the larger pattern shown in Figure 14.

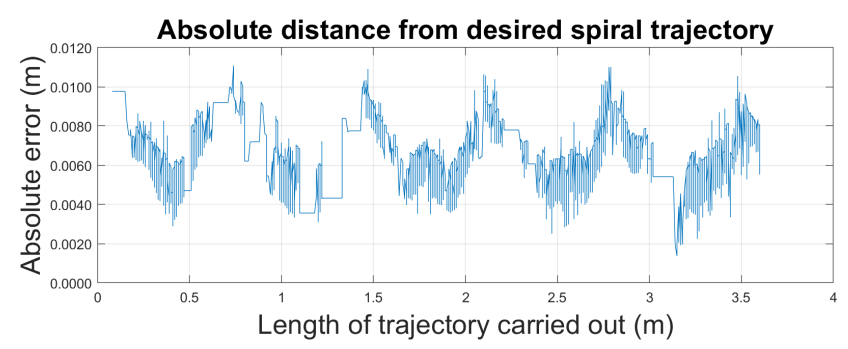

Fig. 11. Absolute error between print trajectory and end effector position for a spiral trajectory

Figure 11 shows that the absolute error never exceeds $0.012 \mathrm{~m}$, which is expected because the distance between each print trajectory pose is $0.01 \mathrm{~m}$. The errors shown here is only due to density of printing trajectory and the printing iiwa arm implementation. Therefore, this experiment establishes the expectation of about $0.015 \mathrm{~m}$ error that should not be violated when simultaneous motion is introduced.

\section{B. Simultaneous motion printing - Ice cream silhouette}

Due to the omni-directionality and high-DoF, the MAP robot system is highly redundant. This allows for many possible strategies and trajectories to be adopted by the mobile platform and printing arm. To investigate this, a fourphase experiment, employing different motion strategies was conducted using a non-standard shape to increase complexity. 
MAP robot was sent a piece-wise continuous, closed loop, roughly Ice cream silhouette shaped printing trajectory made out of four quarters (Labeled Q1, Q2, Q3, Q4 in figure 12 ). The first two quarters (Q1,Q2) printed a $0.05 m$ amplitude wave on a 3 mradius circular arc. However, these quarters illustrate different strategies. Q1 employed the omnidirectionality of the platform as it maintained a $\frac{p i}{4}$ heading, whilst the extruder heading was outwards facing. During Q2, the mobile platform was exercising the position-orientation control to maintain outwards facing heading, whilst end effector heading was a constant $\frac{3 p i}{4}$. For the latter two quarters, printed segments of straight lines, the middle sections of both were with simultaneous velocity whereby the start and end sections involved the platform turning in place as the arm joined the neighboring quarters. In the fourth quarter (Q4) we introduced a $0.05 \mathrm{~m}$ wave into the mobile platform's path. The shape of this print trajectory as well as the data of the experiment is shown in the Figure 12.
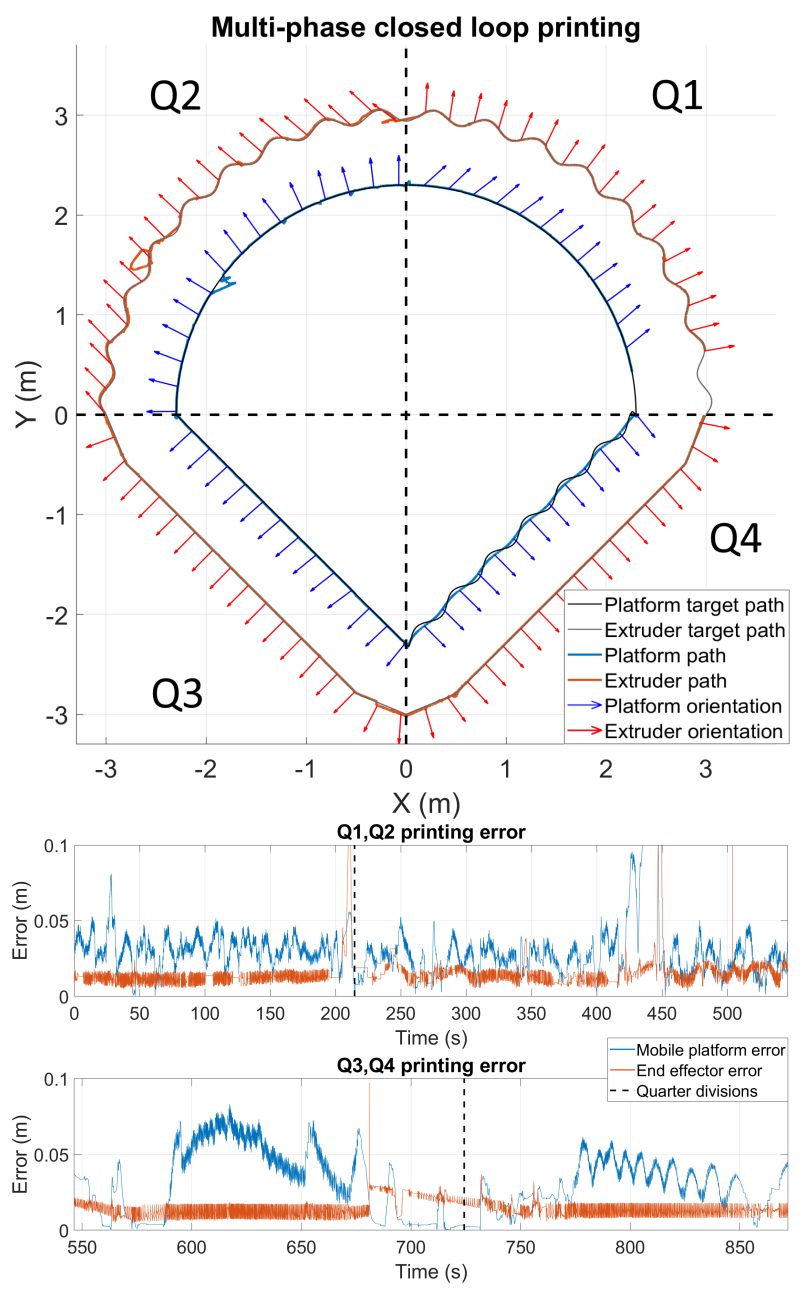

Fig. 12. Multi-phase experiment carrying out a complex print trajectory whilst employing different motion strategies in quarters Q1-4. Top: shows 2D path and heading followed.Bottom: shows the corresponding absolute Cartesian errors for mobile platform and arm end effector-extruder. Note that the start of Q1 data is missing due to a later start in recording the data.

These findings support the notion that simultaneous motion of the printing arm and mobile platform does not lead to reduced printing quality. Throughout the experiment and especially in Q1, the extruder error was within the expected $0.015 \mathrm{~m}$ margin. The end effector error exceeded $0.05 \mathrm{~m}$ between Q1 and Q2, and in Q2. This is a consequence of aggressive mobile platform heading adjustments, where the mobile platform rotated about the vertical axis. This is caused by discrete switching of control mode from translation to orientation between Q1 and Q2, and error in mobile platform orientation exceeding a certain threshold during Q2. The trajectory planning does not cause these errors, the heading adjustments cause the end effector to move faster than the motion compensation can account for, so system stops printing while these heading adjustments occur. The data suggests that maintaining constant platform velocity magnitude allows for the the arm to robustly adapt as it prints.

Furthermore, the data illustrates the print-leading property of the system as in quarters 3 and 4, the mobile platform error is seen to grow. The straight line trajectory requires minor printing arm adjustments and hence is faster to print. The mobile platform does not follow its path in Q4 for the same reason. However, the end effector position is what drives the system to the next set-point, so this is to be expected.

\section{CONCLUSION AND FURTHER WORK}

Having printed a trajectory length approx. 30 times its own diameter, MAP has illustrated the potential of agile, independent, omni-directional robots with high-DoF manipulators can achieve in large-scale 3D printing.

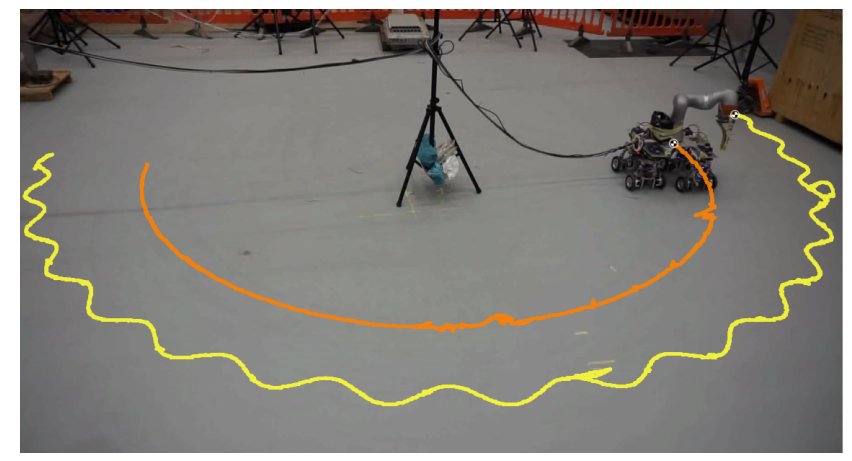

Fig. 13. Time lapse of path taken by the end effector and the mobile platform as the system performs the trajectory shown in Figure 12. Yellow is the path of the printing robot arm and orange is the mobile platform. Path rendered via post processing of the video.

One of the few comparable systems - Digital Construction Platform (DCP)[31] achieves working volume of $2786 \mathrm{~m}^{3}$, and end effector accuracy of $0.06 \mathrm{~mm}$. MAP falls short by these metrics, as current setup allows total working volume of approximately $180 \mathrm{~m}^{3}$, with an average end-effector accuracy of $0.015 \mathrm{~m}$. However,since it displays coordination of printing and motion that scales without loss of accuracy, the working volume of MAP is limited only by the height of the robot and the dimensions of the tracked space. On the other hand the DCP prints from a static base position and has relocation costs.

Whilst the current implementation has allowed us to isolate the effects omni-directional motion on printing function 


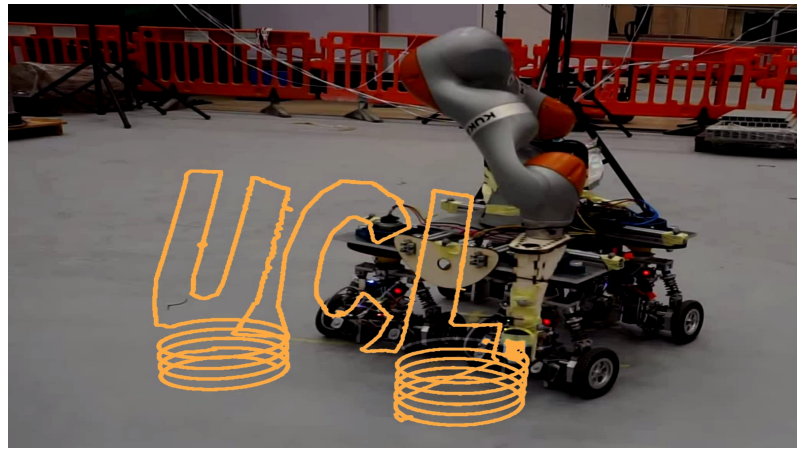

Fig. 14. Time lapse of path taken by the end effector as the system performs a complex $6 \mathrm{DOF}$ trajectory. Path rendered via post processing of the video.

and quality, immediate extension of work would be to replace the Optitrack system by integrating a SLAM system to enable tests within an outdoors environment. To increase the workspace, we will also eliminate the tether with on board power, wireless communication for the printing arm and a material extrusion system mounded on the platform.

By demonstrating high-DoF (Figure 14.), large-scale printing (Figure 13.), MAP opens doors for future investigations in on-site construction robots. These include adapting the platform further for testing when interacting in a dynamic building environments: by evaluating and increasing rough terrain tolerance, introducing a printing trajectory compensation relative to material already deposited, investigating feasibility of holonomic motion and co-working with humanrobot operators. The most exciting of all future extensions are multi-agent or swarm printing of multiple MAP robot platforms and the corresponding autonomous generation of print and platform trajectories, which should be integrated with Computer Aided and Parametric Design work flows.

\section{ACKNOWLEDGEMENT}

The presented work was supported by the Engineering and Physical Sciences Research Council (EP/N018494/1).

\section{REFERENCES}

[1] V. M. Pawar, R. Stuart-Smith, and P. Scully, "Toward autonomous architecture: The convergence of digital design, robotics, and the built environment," Science Robotics, vol. 2, no. 5, p. eaan3686, 2017.

[2] A. Morby, "O’Rourke off-site housing factory gets $£ 22 \mathrm{~m}$ grant,” 2015.

[3] Health and Safety Executive, "Health and safety statistics for the construction sector in Great Britain, 2017," Health and Safety Executive, Crown Copyright, Tech. Rep., 2017.

[4] Z. Dakhli and Z. Lafhaj, "Robotic mechanical design for brick-laying automation," Cogent Engineering, vol. 4, no. 1, p. 1361600, 2017.

[5] M. Vomhof, L. Vasey, S. Brauer, K. Eggenschwiler, J. Strauss, F. Gramazio, and M. Kohler, "Robotic fabrication of acoustic brick walls," 2014

[6] J. Werfel, K. Petersen, and R. Nagpal, "Designing collective behavior in a termite-inspired robot construction team," Science, vol. 343, no. 6172, pp. 754-758, 2014.

[7] N. Michael, J. Fink, and V. Kumar, "Cooperative manipulation and transportation with aerial robots," Autonomous Robots, vol. 30, no. 1, pp. 73-86, 2011

[8] I. Jokic, Sasa, I. Novikov, Petr, I. Shihui, Jin, I. Maggs, Stuart, I. Nan, Cristina, and D. I. Sadan, "Mini Builders," 2017. [Online]. Available: http://robots.iaac.net/\#
[9] J. McLurkin, A. J. Lynch, S. Rixner, T. W. Barr, A. Chou, K. Foster, and S. Bilstein, "A low-cost multi-robot system for research, teaching, and outreach," in Distributed Autonomous Robotic Systems. Springer, 2013, pp. 597-609.

[10] Z. Wang, G. Yang, X. Su, and M. Schwager, "Ouijabots: Omnidirectional robots for cooperative object transport with rotation control using no communication," in International Conference on Distributed Autonomous Robotics Systems (DARS), London, UK, 2016.

[11] J. Klingner, A. Kanakia, N. Farrow, D. Reishus, and N. Correll, "A stick-slip omnidirectional powertrain for low-cost swarm robotics: Mechanism, calibration, and control," in 2014 IEEE/RSJ International Conference on Intelligent Robots and Systems.

[12] T. Aoki, H. Yamato, M. Shimaoka, and S. Mitsumori, "Study of omni-directional all terrain mobile robot with globular metal spring wheel," in Robotics and Automation (ICRA), 2013 IEEE International Conference on. IEEE, 2013, pp. 5606-5611.

[13] J. E. M. Salih, M. Rizon, S. Yaacob, A. H. Adom, and M. R. Mamat, "Designing omni-directional mobile robot with mecanum wheel,' American Journal of Applied Sciences, vol. 3, no. 5, pp. 1831-1835, 2006

[14] G. Ishigami, K. Iagnemma, J. Overholt, and G. Hudas, "Design, development, and mobility evaluation of an omnidirectional mobile robot for rough terrain," Journal of Field Robotics, vol. 32, no. 6, pp 880-896, 2015.

[15] D. Choi, J. R. Kim, S. Cho, S. Jung, and J. Kim, "Rocker-pillar: Design of the rough terrain mobile robot platform with caterpillar tracks and rocker bogie mechanism," in Intelligent Robots and Systems (IROS), 2012 IEEE/RSJ International Conference on. IEEE, 2012.

[16] B. Coxworth and T. Lexen, "Robot rover tackles uneven ground using screw drive," 2017. [Online]. Available: http: / / newatlas.com/

[17] H. Hamann, "Scenarios of swarm robotics," in Swarm Robotics: A Formal Approach. Springer, 2018, pp. 65-93.

[18] M. Udengaard and K. Iagnemma, "Design of a highly maneuverable wheeled mobile robot," in Unmanned Systems Technology X, vol. 6962. International Society for Optics and Photonics, 2008, p. 696219.

[19] M. A. Kreiger, B. A. MacAllister, J. M. Wilhoit, and M. P. Case, "The current state of $3 \mathrm{~d}$ printing for use in construction," in Conference on Autonomous and Robotic Construction of Infrastructure, 2015, pp. 149-157.

[20] B. Sevenson, "Shanghai-based winsun 3d prints 6-story apartment building and an incredible home," 3DPrint. com, 2015.

[21] G. Zhang, X. Li, R. Boca, J. Newkirk, B. Zhang, T. Fuhlbrigge, H. Feng, and N. Hunt, "Use of industrial robots in additive manufacturing - a survey and feasibility study," pp. 512-517, 012014.

[22] J. R. Kubalak, C. D. Mansfield, T. H. Pesek, Z. K. Snow, E. B. Cottiss, O. D. Ebeling-Koning, M. G. Price, M. H. Traverso, L. D. Tichnell, C. B. Williams, et al., "Design and realization of a 6 degree of freedom robotic extrusion platform."

[23] P. Singh and D. Dutta, "Multi-direction slicing for layered manufacturing," Journal of Computing and Information Science in Engineering, vol. 1, no. 2, pp. 129-142, 2001.

[24] S. Keating and N. Oxman, "Compound fabrication: A multi-functional robotic platform for digital design and fabrication," Robotics and Computer-Integrated Manufacturing, vol. 29, no. 6, pp. 439-448, 2013.

[25] 2018. [Online]. Available: http://optitrack.com/

[26] T. B. Park, J. H. Lee, B.-J. Yi, W. K. Kim, B. J. You, and S.R. Oh, "Optimal design and actuator sizing of redundantly actuated omni-directional mobile robots," in Robotics and Automation, 2002. Proceedings. ICRA'02. IEEE International Conference on, vol. 1 IEEE, 2002, pp. 732-737.

[27] KUKA, "Kuka sunrise.os," 2018. [Online]. Available: https: / /www. kuka.com/

[28] W. Meeussen, "Ros control package," 2018. [Online]. Available: https: / / www.wiki.ros.org/ros_control

[29] I. A. Sucan and S. Chitta, "Moveit motion planning framework," ROS, 2018. [Online]. Available: http://moveit.ros.org/about/

[30] J. J. Kuffner and S. M. LaValle, "Rrt-connect: An efficient approach to single-query path planning," in Proceedings 2000 ICRA. Millennium Conference. IEEE International Conference on Robotics and Automation. Symposia Proceedings (Cat. No.00CH37065), vol. 2, 2000, pp 995-1001 vol.2.

[31] S. Keating, J. C. Leland, L. Cai, and N. Oxman, "Toward site-specific and self-sufficient robotic fabrication on architectural scales," vol. 2 , p. eaam8986, 042017. 